\title{
L'HOMME L'Homme
}

Revue française d'anthropologie

157 | janvier-mars 2001

Représentations et temporalités

\section{Patricia Jeffery \& Amrita Basu, eds., Appropriating Gender. Women's Activism and Politicized Religion in South Asia}

New York-London, Routledge, 1998, XII + 276 p., bibl., index (« Zones of Religion »)

\section{Véronique Bénéï}

\section{(2) OpenEdition}

\section{Journals}

Édition électronique

URL : http://journals.openedition.org//homme/5861

DOI : 10.4000/lhomme.5861

ISSN : 1953-8103

Éditeur

Éditions de l'EHESS

Édition imprimée

Date de publication : 1 janvier 2001

Pagination : 309-312

ISBN : 2-7132-1357-6

ISSN : 0439-4216

Référence électronique

Véronique Bénéï, « Patricia Jeffery \& Amrita Basu, eds., Appropriating Gender. Women's Activism and Politicized Religion in South Asia », L'Homme [En ligne], 157 | janvier-mars 2001, mis en ligne le 23 mai 2007, consulté le 25 septembre 2020. URL : http://journals.openedition.org/lhomme/5861 ; DOI : https://doi.org/10.4000/lhomme.5861

Ce document a été généré automatiquement le 25 septembre 2020

(c) École des hautes études en sciences sociales 


\section{Patricia Jeffery \& Amrita Basu, eds., Appropriating Gender. Women's Activism and Politicized Religion in South Asia}

New York-London, Routledge, 1998, XII + 276 p., bibl., index (« Zones of Religion »)

Véronique Bénéï

1 NUL N'IGNORE plus l'essor des mouvements religieux et politiques qu'a connu l'Asie du Sud au cours de cette dernière décennie. Les courants islamistes dans tout le souscontinent indien, comme les partis de la droite hindoue en Inde, ont investi la sphère publique pour la travailler conformément à leurs idéaux; tantôt sur le mode autoritaire, au Pakistan par exemple, tantôt sur le mode démocratique, en Inde. On méconnaît davantage la redéfinition des « rapports sociaux de sexe » qui l'accompagna, et l'utilisation qu'en firent ces mêmes mouvements. On méconnaît plus encore le rôle qu'y jouèrent les femmes. Pas seulement les activistes féministes, mais aussi les femmes ordinaires. Jusqu'à présent, celles-ci avaient été considérées comme des reproductrices biologiques et sociales cantonnées à la sphère domestique. Mais, d'une part, leur quotidien demeurait largement inexploré dans les études des mouvements religieux et politiques; d'autre part, leur participation accrue dans les mouvements nationalistes d'extrême droite demeurait secondaire aux yeux des observateurs de tout bord, féministes comprises. Or, ce qui s'est passé le 6 décembre 1992 en Inde du Nord, à Ayodhya, ville située dans l'État d'Uttar Pradesh, fit l'effet d'un détonateur. Ce jour-là, plusieurs milliers de militants nationalistes hindous démolirent la mosquée de Babri, vieille de quatre siècles. Les encourageaient leurs "sœurs" hindoues, lesquelles chantaient frénétiquement et les exhortaient à tuer le musulman. On comprit alors pour la première fois que les femmes, d'habituels objets de la violence domestique et communautaire, pouvaient aussi se transformer en sujets, actrices de la violence. 
2 Depuis, une réflexion s'est amorcée sur la participation des femmes aux mouvements religieux et nationalistes en Asie du Sud. Un ouvrage pionnier a vu le jour en 1995, publié par la première maison d'édition féministe, Kali for Women : exclusivement consacré à l'Inde et essentiellement aux femmes hindoues, l'ouvrage dirigé par Tanika Sarkar et Urvashi Butalia, Women and Right-Wing Movements. Indian Experiences ${ }^{1}$, présentait des réflexions de féministes, des témoignages d'avocates spécialisées dans le droit des femmes, le rapport d'une délégation de partis politiques de gauche sur les violences commises à l'encontre des femmes appartenant aux minorités dans plusieurs villes indiennes, des interviews de leaders féminins de mouvements de la droite hindoue, ainsi que des travaux portant, par exemple, sur la construction des stéréotypes de l'hindou et du musulman à l'époque de la partition, ou sur la légitimation du viol comme arme politique. Ce livre permettait de comprendre «de l'intérieur » l'engouement pour le mouvement hindutva des femmes issues des classes moyennes et populaires. D'autres lui ont fait suite, avec plus ou moins de bonheur.

3 Le présent volume est assurément un pas de plus dans l'étude des rapports entre sexe, religion et politique en Asie du Sud. Issu pour partie d'un colloque qui s'est tenu au mois de septembre 1994 au Rockefeller Center de Bellagio, en Italie, il rassemble les contributions de treize universitaires. Son envergure internationale transparait tant dans le choix des contributeurs que par sa perspective comparative. Les treize contributeurs sont pour moitié originaires d'Asie du Sud, pour moitié anglais ou américains. Du Pakistan au Sri Lanka en passant par l'Inde et le Bangladesh, c'est la totalité de l'Asie du Sud qui est couverte. On y aborde aussi bien le point de vue de musulmanes que d'hindoues, et plus encore la question des rapports sociaux de sexe là où les musulmans sont majoritaires dans le sous-continent (Pakistan, Bangladesh), et non plus dans le seul contexte indien où les adeptes de l'islam ne représentent « qu' »une minorité.

4 Centrale est ici la notion d'agency - que l'on pourrait rendre par action sociale - d'une personne, d'un groupe ou d'une institution. En explorant les diverses formes qu'elle revêt tant sur le plan de l'État que sur ceux de la vie quotidienne et de l'engagement politique, les auteurs analysent la manière dont les femmes elles-mêmes construisent leurs identités individuelles et collectives (Amrita Basu). Le livre se compose de trois parties rassemblant respectivement six, trois et quatre chapitres (introduction et conclusion comprises). La première traite de l'influence de l'État et des communautés religieuses sur la construction des identités sexuées; la deu-xième examine, au moyen d'exemples précis, la question de la religion vécue au quotidien par les femmes; la troisième étudie l'action sociale des femmes au sein de - ou contre les - mouvements « religieux et ethniques» (sic).

5 C'est à l'islam au Bangladesh (Shelley Feldman), au Pakistan (Shahnaz Rouse) et en Inde (Zoya Hasan) que sont consacrées la moitié des contributions de la première partie. Shahnaz Rouse et Shelley Feldman montrent respectivement comment, à la faveur de la politique de développement économique qui a immédiatement suivi l'indépendance du Pakistan, puis, plus tard, du Bangladesh, les femmes - y compris celles qui appartenaient aux secteurs les moins favorisés de la population, urbaine comme rurale - ont eu accès à des emplois publics qui leur ont permis de sortir de la sphère domestique. Au Bangladesh, ce phénomène s'est accompagné de la constitution progressive d'une identité bangladeshie combinant des éléments culturels - bengalis et religieux - islamiques. Dans les deux cas cependant, l'affirmation d'un régime 
politique fort dans les décennies suivantes est allée de pair avec l'accentuation d'une revendication religieuse où les courants islamistes ont joué un rôle de premier plan. Le résultat s'est souvent traduit par de nouvelles restrictions pour les femmes. Au Pakistan, les réformes juridiques successives ont eu pour effet - sinon pour objet principal de limiter leur mobilité tant spatiale que sociale, voire de légitimer la violence sexuelle exercée à leur encontre. La situation qui prévaut en Inde est différente: l'invisibilité économique de la majorité des femmes d'obédience islamique est inversement proportionnelle à leur visibilité politique en tant qu'enjeu de revendications sécularistes. La question des droits juridiques séparés pour les femmes musulmanes a été en effet un des thèmes principaux lors des débats sur le sécularisme. Non seulement elle a renforcé l'idée qu'il existe dans la société indienne deux communautés différentes - les hindous et les musulmans -, mais elle a créé un espace politique distinct pour les musulmans, qui a lui-même contribué à leur exclusion des transformations sociales et économiques de plus grande ampleur. Notons qu'elle a en outre servi la droite politique hindoue, puisque celle-ci s'est présentée, en contraste avec les musulmans conservateurs, comme championne de l'égalité des femmes et comme laïque par son souhait d'un code civil uniforme indien.

6 Tanika Sarkar retrace utilement la trajectoire historique d'une politique de l'identité hindoue élaborée dès la fin du XIX ${ }^{e}$ siècle par les réformateurs sociaux. Elle rappelle que les idéologues successifs de ces mouvements se mobilisèrent autour de la question de la femme pour (re)définir une communauté hindoue au-delà de l'appartenance de caste. Elle étudie également les premières phases de l'engagement politique des femmes hindoues au début du siècle, et notamment la création de branches féminines dans les partis représentant cette droite religieuse. Les activités pédagogiques y étaient prépondérantes. Tanika Sarkar évoque en passant l'objectif de la fondatrice de l'une de ces branches : permettre aux femmes, par un apprentissage physique, de se défendre contre la violence masculine, y compris hindoue. L'auteur souligne aussi l'émergence plus récente, dans les rapports conflictuels entre hindous et musulmans, de l'image puissamment évocatrice de la femme hindoue agressée par le musulman.

7 Ritu Menon revient sur l'événement traumatique de la partition en analysant comment le corps de ces femmes enlevées en Inde et au Pakistan devint fondamental dans la légitimation tant de la communauté hindoue en Inde que de l'État indien nouvellement créé. Le corps des femmes est un marqueur à la fois de frontières territoriales, de communauté et de sexe (au sens de "gender »). Dans cette lutte pour la légitimation, l'État indien fut lui-même l'auteur de violences physiques - par le truchement des travailleuses sociales et des enquêteurs chargés de retrouver les femmes enlevées - en réintégrant par la force celles qui refusaient de revenir dans leur ancienne famille.

La deuxième partie porte sur le « quotidien et le local ». Il est regrettable qu'elle ne soit pas aussi fournie que la précédente, car ce sont les études sur les femmes dans leur vie de tous les jours qui font le plus défaut - en Asie du Sud, mais pas seulement -, alors même qu'elles conféreraient une dimension supplémentaire à l'étude de l'activisme politique et religieux féminin. Prenant le Pakistan pour exemple, Farida Shaheed dénonce l'amalgame souvent effectué entre les expériences personnelles des acteurs sociaux et l'usage politique qui est fait de la religion dans la sphère publique. Se demandant pourquoi la religion est devenue aujourd'hui l'idiome politique privilégié en Asie du Sud, elle note la place centrale qu'y occupent les définitions des rôles sexués. Les récits de femmes qu'elle a recueillis disent la difficulté qu'éprouvent celles-ci à 
remettre en cause ces définitions, que véhiculent les membres de leur famille et qui les marquent au plus intime de leur quotidien. Mais, l'auteur a raison d'y insister, cette difficulté n'est ni vécue ni pensée sur le mode de la religion. Au contraire, ces femmes analysent leur situation en termes de "culture traditionnelle ", de normes sociétales et de contrôle masculin.

9 Patricia Jeffery et Roger Jeffery insistent eux aussi sur l'importance du «sexisme routinisé » (sic) dans la vie domestique ordinaire des femmes. Mais, dans le district de Bijnor, situé dans l'Uttar Pradesh, qui compte une majorité de musulmans, les distinctions de sexe ne sont pas seulement les marqueurs de l'« identité musulmane »: elles jouent un rôle non moins considérable dans les conflits « communalistes » entre hindous et musulmans, au même titre que les distinctions de classe. Dans ce district, les instances de l'État (éducatives, sanitaires, administratives, etc.) favorisent et renforcent les perceptions communautaires en réservant leur accès aux hindous au détriment des musulmans.

10 Par contraste, Barbara Metcalf, qui se penche sur l'engagement récent des femmes dans le mouvement religieux islamiste de la Tablîghî Jama'at en Asie du Sud, offre une perspective idéalisée dans laquelle toutes les distinctions - de statut, de sexe, etc. - sont abolies. Pour autant, les femmes ne sont présentes que de manière périphérique. Certes, elles ont aussi leur "tournée ", élément capital dans le mouvement de la Tablîghî: les dévots font un voyage qui peut durer de quelques jours à plusieurs mois. Mais celui-ci ne concerne qu'une infime proportion d'entre elles, tandis que la majorité demeure à la place que leur assigne leur sexe, à savoir le foyer domestique.

11 Dans la troisième partie, Amrita Basu s'intéresse au rôle actif que les femmes ont joué au cours de cette dernière décennie dans la violence perpétrée par les partis de l'extrême droite hindoue. Elle relève que la participation à la violence communautaire n'est pas uniquement le fait de quelques égéries de ces partis, célèbres pour leurs exhortations envers leurs « frères hindous » à " se conduire en hommes » - c'est-à-dire à occire l'ennemi, le musulman -, en telle ou telle occasion. Malheureusement, l'auteur perd de vue son objet pour s'attarder sur les distinctions entre communalisme et fondamentalisme, sur lesquelles peu d'auteurs s'accordent. Les femmes n'y apparaissent plus que comme les otages de débats politiques dans lesquels non seulement leurs véritables intérêts importent peu, mais où elles n'ont que rarement voix au chapitre.

12 En revanche, Malathi de Alwis montre comment, dans le conflit qui opposa, de 1987 à 1991, l'État du Sri Lanka au Parti Nationaliste des Jeunes Sinhalais, alors en plein renouveau, des mères s'inspirèrent du mouvement des Mères d'Argentine pour lutter contre l'État avec les seules armes que leur conférait le statut social de la maternité. Plus de soixante mille jeunes gens et hommes d'âge moyen affiliés au Parti Nationaliste, enlevés par les sbires du gouvernement, avaient été portés disparus. Ces femmes manifestèrent tant et plus contre l'État en leur qualité de mères et/ou d'épouses. Dans cette bataille assidue - qui finit d'ailleurs par être récupérée politiquement par des leaders hommes du Parti de la Liberté du Sri Lanka -, les larmes, les cris et les rituels religieux furent autant de registres sur lesquels les mères des disparus déclinèrent leur protestation publique, obligeant le chef de l'État à répondre en termes identiques (par exemple en organisant un « contre-rituel » de masse).

13 En conclusion, Patricia Jeffery s'interroge sur les implications de la notion d'« agency » et sur sa valeur heuristique, s'agissant des études sur les femmes en Asie du Sud. Elle 
pose la question de savoir quelle sorte d'agents sociaux peuvent être les femmes, compte tenu de leur subordination. Le problème est précisément qu'elles restent subordonnées. En ce sens, si le propos initial de l'ouvrage était de réfléchir sur la manière dont les femmes elles-mêmes "strategically appropriated gender to achieve social change » (p.3), il reste que, hormis dans quelques contributions, ces femmes d'Asie du Sud, qu'elles vivent au Bangladesh, au Sri Lanka, au Pakistan ou en Inde, qu'elles soient musulmanes ou hindoues, qu'elles appartiennent à la majorité ou à la minorité, apparaissent trop rarement en tant qu'actrices sociales autonomes. À travers le traitement qui est fait de leurs affiliations politiques, domestiques, sociales, de leurs choix ou non-choix, s'impose un constat décourageant : elles sont encore trop souvent considérées comme objets et non comme sujets sociaux, y compris parfois par ceux et celles qui s'efforcent de leur donner la parole.

L'apport du livre n'en demeure pas moins précieux : en mettant en relief la diversité des expériences féminines, y compris de femmes partageant une même religion, notamment dans le cas de l'islam, il travaille contre la simplification. Grâce à leurs points de vue contrastés, l'ensemble des contributions brossent un tableau nuancé et subtil des rapports entre sexe (au sens de «gender»), religion et politique en Asie du sud.

\title{
NOTES
}

1. New Delhi-London, Kali for Women/Zed Books, 1995.

\author{
AUTEUR \\ VÉRONIQUE BÉNÉï \\ CNRS, Maison française d'Oxford, Grande-Bretagne.
}

